\title{
Aqueous Cannabis Extract Prevents Ethylene Glycol-induced Renal Calcium Crystallization
}

\author{
Ahmad Amouzeshi', Seyedeh Zahra Moossavi², Seyed Yoosef Javad \\ Moosavi $^{3}$, Mahmoud Zardast ${ }^{4}$, Mohammad Malekaneh ${ }^{4}$, Sajad Esmaili ${ }^{3}$, \\ Abdolghader Taneh ${ }^{3}$, Nasim Lotfi ${ }^{4}$, Maryam Moossavi ${ }^{3}$, and Reyhane \\ Hoshyar 4,5 \\ ${ }^{1}$ Department of Cardiovascular Surgery, Surgery and Trauma Research Group, Birjand University \\ of Medical Sciences, Birjand, Iran \\ ${ }^{2}$ Medical School, Shiraz University of Medical Sciences, Shiraz, Iran \\ ${ }^{3}$ Student Research Committee, Birjand University of Medical Sciences, Birjand, Iran \\ ${ }^{4}$ Cellular and Molecular Research Center, Birjand University of Medical Sciences, Birjand, Iran \\ ${ }^{5}$ Microbiology and Molecular Genetics, Michigan State University, East Lansing, MI, USA
}

Corresponding Author:

Reyhane Hoshyar

Microbiology and Molecular

Genetics, Michigan State

University, East Lansing, MI

48824, USA

email: rey-

haneh.houshyar@gmail.com

hooshyar@bums.ac.ir

Production and Hosting by

Knowledge E

(c) Ahmad Amouzeshi

et al. This article is distributed

under the terms of the

Creative Commons

Attribution License, which

permits unrestricted use and

redistribution provided that

the original author and source

are credited.

Editor-in-Chief:

Dr. Alireza Rafiei

\section{Abstract}

Background: Some plant extracts can prevent kidney stone formation in a dosedependent manner. In our study, we aimed to investigate the protective role of Cannabis sativa aqueous extraction on calcium oxalate formation in ethylene glycolinduced rats.

Materials and Methods: To evaluate anti-urolithiasis, variations of the main risk factors (citrate, phosphorous, calcium, and $\mathrm{Ph}$ ) have been evaluated in 24-hour urine samples of rats one day before the end of the experiment. Rats were divided into 4 groups: group 1 was administered regular drinking water; group 2 was administered 1\% ethylene glycol in drinking water, group 3 was administered $100 \mathrm{mg} / \mathrm{kg}$ of Cannabis sativa extract for oral administration by gavage along with drinking water containing $1 \%$ ethylene glycol; and group 4 was administered $200 \mathrm{mg} / \mathrm{kg}$ of Cannabis sativa extract by gavage along with drinking water containing $1 \%$ ethylene glycol. Finally, histopathological slides from the kidney were also evaluated.

Results: Results showed that $100 \mathrm{mg} / \mathrm{kg}$ and $200 \mathrm{mg} / \mathrm{kg}$ doses of Cannabis sativa extract significantly reduced the mentioned risk factors in comparison with ethylene glycol-treated rats $(p<0.05)$.

Conclusion: Histopathological slides showed reduced calcifications with extract treatment at $200 \mathrm{mg} / \mathrm{kg}$ of Cannabis sativa. Thus, this antioxidant herb can prevent kidney stone formation. These findings pave the way for new therapy.

Keywords: Antioxidants, Cannabis sativa, Ethylene Glycol, Rats, Urate calcium oxalate stone,

\section{Introduction}

\section{G OPEN ACCESS}

Urinary calculi disease is well-known as nephrolithiasis, and regarded as systemic, painful, and costly, and especially affects the male gender (1). The increased prevalence 
of this disease is pandemic and differs between ethnicity and race. Epidemiological studies revealed the rising prevalence rates globally (2). In our country, the Iran Islamic Republic, the prevalence rate of urolithiasis is $5.7 \%$ (3). Several studies have mentioned many extrinsic (diet and lifestyle), and intrinsic (genetics) causes related to this disease in different individuals. Nephrolithiasis is also associated with different diseases including; chronic kidney disease (CKD), coronary artery/heart disease (CHD), hypertension, type 2 diabetes mellitus (T2DM), and other metabolic syndromes (4). Worldwide, approximately $80 \%$ of kidney stones are composed of calcium oxalate mixed with a small percentage of calcium phosphate (1). Remarkable advances in the therapy and management of individuals with kidney stones have emerged. Shockwave lithotripsy fragments kidney stones to pass through the urine, or percutaneous nephrolithotomy and retrograde intrarenal surgery remove them $(5,6)$. However, these methods are expensive, and in many cases, kidney stones are reversible. Thus, increasingly, research attention has been devoted to medicinal plants as novel and alternative options with effective properties and less cost (7-9). In a similar vein, many herbs such as the aqueous extract of Bergenia ciliate (10) and Chenopodium album (1), have been examined in-vitro for their inhibitory effects on calcium oxalate crystallization. In a recent study, we aim to investigate the role of Cannabis sativa, as an alternative for inhibition of kidney stone formation. Cannabis sativa, famous as marijuana, is probably the world's most recognizable and notorious plant which is harvested chiefly for its fiber (11). It has a massive variety of antioxidant chemicals such as cannabinoids and C-21 terpenophenolic compounds (12). Cannabinoids are highly lipophilic, and when inhaled, can infiltrate cell membranes and cross the blood-brain barrier (BBB). The chief metabolite, 11-hydroxy- $\delta 9 T H C$, is possibly responsible for the psychological and physiological effects of cannabis, such as its regulatory role in feeding behavior, analgesia, mood modulation, anti-inflammatory activity, anti-oxidant activity, energy metabolism, among others (13). Therefore, we decided to investigate the protective effect of Cannabis sativa as a herbal therapy and a less expensive treatment for preventing renal stone formation in a male rat animal model.

\section{Materials and Methods}

\subsection{Plant extracts}

Cannabis sativa leaves were collected and dried under conditions without exposure to direct sunlight and then made into powder. The powder was dissolved in boiling distilled 
water for 30 minutes, then filtered by a sterile filter $(0.2 \mu \mathrm{m})$ and lyophilized for 24 hours (14).

\subsection{Animal care}

In our study, 32 adult male Wistar rats weighing 120-180 $\pm 20 \mathrm{~g}$ was obtained from Birjand University of Medical Sciences, Iran. In all procedures, animal experiments were conducted in accordance with the institutional ethics committee for the care and use of laboratory animals of this university. In all experiments, rats were fed standard rat chow daily and allowed free access to water. Animals were housed individually at $25^{\circ} \pm 3^{\circ} \mathrm{C}$ with a $12 \mathrm{~h}$ light/dark cycle and $50 \% \pm 5 \%$ humidity.

\subsection{Experimental design}

The rats were housed under standard conditions and randomly divided into four groups ( $n=$ eight rats per group). An ethylene glycol diet was used to induce kidney stone formation in rats. The experimental groups were as follows:

Group 1 (normal control): regular rat drinking water ad libitum daily for a period of 28 days; Group 2: 1\% ethylene glycol (Merck, Germany) in drinking water for 28 days; Group 3: a dose of $100 \mathrm{mg} / \mathrm{kg}$ of Cannabis sativa extract for oral administration by gavage along with drinking water containing $1 \%$ ethylene glycol for 28 days; Group 4: a dose of $200 \mathrm{mg} / \mathrm{kg}$ of Cannabis sativa extract by gavage, along with drinking water containing $1 \%$ ethylene glycol for 28 days.

\subsection{Biochemical analysis}

One day before the end of the experiment, the 24-hour urine samples of rats were collected using metabolic cages. The urine $\mathrm{pH}$, oxalate, citrate, calcium, and phosphorous levels were determined using commercially available assay kits $(15,16)$. At the end of the experiment, blood was collected from the aorta under anesthesia, and the animals were sacrificed. The serum was separated for the analysis of creatinine, uric acid, calcium, and phosphorous levels using an auto-analyzer (Technicon RA-1000). 


\subsection{Histopathological examination}

At the end of the experiment, both rat kidneys were used for histopathological analyses and fixed in 10\% buffered formalin and blocked using paraffin wax. After these procedures, they were sectioned at a $5-\mu \mathrm{m}$ thickness and mounted on slides. Then, the sections were stained with hematoxylin and eosin for examination under a polarized light microscope. Furthermore, the numbers of calcium oxalate crystal depositions were calculated using a light microscope fitted with a polarizing filter.

\subsection{Statistics}

Data are presented as the means \pm standard division (SD) and were analyzed using oneway analysis of variance (ANOVA) with SPSS software (SPSS version 15 Inc., Chicago, IL, USA). Bonferroni multiple comparison analysis was applied to compare the differences between the groups. A $P$-value of 0.05 or less was considered statistically significant.

\section{Results}

\subsection{Effects of Cannabis sativa on urine factors}

During our study, Group 1 (normal control): plain drinking water ad libitum daily for 28 days; Group 2: 1\% ethylene glycol in drinking water for 28 days; Group 3: $100 \mathrm{mg} / \mathrm{kg}$ of a Cannabis sativa extract by gavage along with drinking water containing $1 \%$ ethylene glycol for 28 days; Group 4: 200 mg / kg of Cannabis sativa extract by gavage along with drinking water containing $1 \%$ ethylene glycol for 28 days, the urinary $\mathrm{pH}$ remained constant in all groups. Furthermore, the phosphorus, calcium and oxalate levels of the urine were remarkably decreased in groups treated with $100 \mathrm{mg} / \mathrm{kg}$ and $200 \mathrm{mg} / \mathrm{kg}$ doses of Cannabis sativa extract in comparison with the ethylene glycol-induced group. These reductions in levels of phosphorus, calcium, and oxalate were statistically significant $(P<0.001)$, and they gradually returned to normal levels (data not shown). The observed reduction in phosphate, calcium, and oxalate levels was dose-dependent. Additionally, a considerable dose-dependent increase in urine citrate could be observed in groups 3 and 4 , treated with Cannabis sativa extracts, in comparison with group 2 to reach normal levels $(P<0.05)$ (Table 1). 
TABLE 1: Urine analysis of different experimental groups.

\begin{tabular}{|l|c|c|c|c|} 
& Group 1 & Group 2 & Group 3 & Group 4 \\
\hline $\begin{array}{l}\text { Urine phosphorus } \\
\text { (mg/dl) }\end{array}$ & $61.75 \pm 2.11$ & $88.68 \pm 1.15$ & $73.35 \pm 1.19^{*}$ & $64.11 \pm 1.11^{*}$ \\
\hline Urine calcium (mg/dl) & $6.38 \pm 0.76$ & $11.15 \pm 0.84$ & $7.31 \pm 1.11^{*}$ & $6.16 \pm 0.08^{*}$ \\
\hline Urine oxalate (mg/dl) & $17.15 \pm 1.12$ & $33.06 \pm 2.02$ & $24.11 \pm 1.18^{*}$ & $20.16 \pm 2.06^{*}$ \\
\hline Urine citrate (mg/dl) & $21.55 \pm 1.71$ & $17.21 \pm 1.15$ & $19.84 \pm 1.22^{*}$ & $20.59 \pm 1.12^{*}$ \\
\hline Urine pH & $6.51 \pm 0.52$ & $6.16 \pm 0.44$ & $6.52 \pm 0.31$ & $6.62 \pm 0.28$ \\
\hline${ }^{*}<<0.05$ compared with group 2 & & & \\
\hline
\end{tabular}

\subsection{Effects of Cannabis sativa on serum factors}

As shown in Table 2, Group 1 (normal control): plain drinking water ad libitum daily for 28 days, Group 2: $1 \%$ ethylene glycol in drinking water for 28 days, Group 3: 100 mg/kg of Cannabis sativa extract by gavage, along with drinking water containing $1 \%$ ethylene glycol for 28 days; Group 4: 200 mg/kg of Cannabis sativa extract by gavage along with drinking water containing $1 \%$ ethylene glycol for 28 days, the serum calcium levels in both treated groups are statistically increased in comparison with the ethylene glycol-induced group $(p<0.05)$. It seems that the treated groups return to normal values gradually. There was no apparent difference in serum phosphorus levels between treated groups and both controls. Serum uric acid concentration and creatinine analysis in groups 3 and 4 became notably lower in comparison with group $2(P<0.05)$.

TABLE 2: Serum analysis from different groups.

Serum calcium $(\mathrm{mg} / \mathrm{dl})$
Serum phosphorus $(\mathrm{mg} / \mathrm{dl})$
Serum Uric acid $(\mathrm{mg} / \mathrm{dl})$
Serum Creatinine $(\mathrm{mg} / \mathrm{dl})$

\begin{tabular}{|c|}
\hline Group 1 \\
\hline $8.88 \pm 0.33$ \\
\hline $8.05 \pm 0.88$ \\
\hline $3.73 \pm 0.23$ \\
\hline $0.68 \pm 0.11$ \\
\hline
\end{tabular}

\begin{tabular}{c} 
Group 2 \\
\hline $6.21 \pm 0.83$ \\
\hline $8.56 \pm 0.63$ \\
$9.22 \pm 1.01$ \\
$1.6 \pm 0.15$
\end{tabular}

\begin{tabular}{|c|}
\hline Group 3 \\
\hline $7.21 \pm 0.26^{*}$ \\
\hline $8.92 \pm 0.57$ \\
\hline $4.45 \pm 0.55^{*}$ \\
\hline $0.84 \pm 0.13^{*}$ \\
\hline
\end{tabular}
Group 4 $7.67 \pm 0.35^{*}$ $8.98 \pm 0.45$ $3.12 \pm 0.68^{*}$ $0.71 \pm 0.15^{*}$

${ }^{*} \mathrm{P}<0.05$ compared with group 2

\subsection{Effects of Cannabis sativa on histopathologic factors}

The number of calcium oxalate crystals were remarkably reduced in a dose-dependent manner (Table 3). Group 1 (normal control): regular drinking water ad libitum daily for 28 days; Group 2: 1\% ethylene glycol in drinking water for 28 days; Group 3: 100 mg/kg of Cannabis sativa extract by gavage along with drinking water containing $1 \%$ ethylene glycol for 28 days; Group 4: 200 mg/kg of Cannabis sativa extract by gavage along with 
drinking water containing $1 \%$ ethylene glycol for 28 days. Furthermore, the histopathological features of the renal tissue related to healthy control rats manifested regular characteristics without signs of calcium oxalate deposits (Figure 1-A). Figure 1-B shows renal segments of ethylene glycol-induced control rats with full signs of calcium oxalate deposits. The number of calcium oxalate deposits in the kidney slides of rats receiving a $200 \mathrm{mg} / \mathrm{kg}$ dose of Cannabis sativa extract were remarkably reduced in comparison with rats administered a $100 \mathrm{mg} / \mathrm{kg}$ dose (Figure 1-C\&D). Calcium oxalate crystals in various parts of the renal tissue in group 4 were considerably smaller in comparison with group 3.

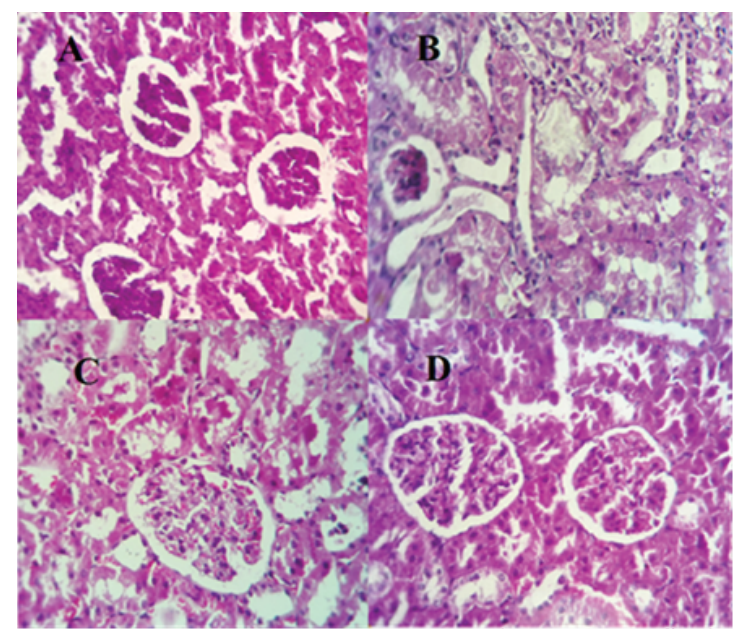

Figure 1: Kidneys histopathologic slides; $A=$ Group 1 (normal control): regular rat drinking water ad libitum daily in a period of 28 days, B=Group 2: 1\% ethylene glycol (Merk, Germany) in drinking water for 28 days, $\mathrm{C}=$ Group 3: dose of $100 \mathrm{mg} / \mathrm{kg}$ of cannabis sativa extract for oral administration by gavage along with drinking water containing $1 \%$ ethylene glycol for 28 days, D=Group 4: dose of $200 \mathrm{mg} / \mathrm{kg}$ of cannabis sativa extract by gavage along with drinking water containing $1 \%$ ethylene glycol for 28 days.Microscopic magnification:40x.

TABLE 3: The number of calcium oxalate crystals formed in different groups.

\begin{tabular}{l|c|c|c|c|} 
& Group 1 & Group 2 & Group 3 & Group 4 \\
\hline $\begin{array}{l}\text { Number of calcium } \\
\text { oxalate crystals }\end{array}$ & 0 & $28 \pm 3.5$ & $8 \pm 15^{*}$ & $2.5 \pm 1.1^{*}$ \\
\hline${ }^{*}<<0.05$ compared with group 2. & & & \\
\hline Microscopic field $=10$ & & & \\
\hline Magnification $=40$ & & &
\end{tabular}

\section{Discussion}

Urinary calculi cause severe morbidity worldwide, and surgical interventions have brought about serious side-effects (17). In this regard, new therapies with fewer complications, for example, phytotherapy as a favorable alternative approach are of significant 
research interest (7). In our study, we investigated the preventive effects of Cannabis sativa aqueous extracts on calcium oxalate formation in ethylene glycol-induced male rats. Rats were used to mimic urolithiasis -kidney calcium oxalate deposition- by ethylene glycol induction due to the similarity of their urinary system to humans (18). In the primary stage of renal stone formation, over-saturation of the urine is accomplished with components, notably calcium and oxalate. Oxidative stress speeded the process of crystallization and aggregation, which results in stone formation (19). Although the process of urinary calcification is multi-factorial, studies have demonstrated the functional role of oxygen free radicals during the development of cellular injuries. Therefore, these injuries support the events of calcium oxalate maintenance in kidney tubules and ultimately, stone development (20).

Conversely, citrate inhibits the aggregation of $\mathrm{CaOx}$ crystals on the renal epithelial cell membrane (21). Several studies suggest that the non-cannabinoid fraction of the natural extract from Cannabis sativa can scavenge free radicals (22); hence, one mechanism by which aqueous Cannabis sativa extracts prevent $\mathrm{CaOx}$ stone formation and aggregation may be by their antioxidant components. Since oxalate is a chief factor in $\mathrm{CaOx}$ stone formation and citrate is regarded as an efficient suppressor of $\mathrm{CaOx}$ stone aggregation, it seems noteworthy to suggest Cannabis sativa plant extracts as a novel remedy for urolithiasis. We observed increased urinary phosphorus, calcium, and oxalate levels and a decrease in citrate levels in ethylene glycol-treated rats. Increased excretion of the mentioned factors has been reported in stone formation and this provides a suitable environment for crystallization (23).

Administration of $100 \mathrm{mg} / \mathrm{kg}$ and $200 \mathrm{mg} / \mathrm{kg}$ doses of Cannabis sativa extracts to groups 3 and 4 reduced crystallization factor levels in a dose-dependent manner, reverting almost to normal control levels. Furthermore, the citrate level was reduced in ethylene glycol-treated rats, in which Cannabis sativa extract prevented over-saturation with citrate in treated groups in a dose-dependent manner. The histopathologic analysis and calcium oxalate crystal reduction confirmed our findings. We observed that the number of calcium oxalate deposits in the kidney slides of rats receiving $100 \mathrm{mg} / \mathrm{kg}$ and $200 \mathrm{mg} /$ $\mathrm{kg}$ doses of Cannabis sativa extracts, in comparison with ethylene glycol-treated rats were remarkably and dose-dependently reduced. Inconsistent with our study, Alireza Golshan et al. reported that $200 \mathrm{mg} / \mathrm{kg}$ of a Cynodon dactylon extract, a medicinal plant with anti-oxidant properties similar to Cannabis sativa, can reduce stone formation in rats receiving ethylene glycol (1\%) in drinking water (24).

Furthermore, another study indicated that the antioxidant activity of Dolichos biflorus makes it a useful plant for therapy of kidney stones and urinary disorders. Treatment of 
ethylene glycol-treated rats with a hydro-alcoholic extract of its seeds reduced calcifications in the kidneys (25). Furthermore, Bergenia ciliata, well-known for its anti-oxidant properties, is used in traditional medicine for treating renal disorders, including kidney stones. This study also showed reduced calcifications following extract treatment (26). In conclusion, these data indicate that a Cannabis sativa extract can prevent crystallization in urolithiasis rats. Therefore, aqueous Cannabis sativa extracts may be candidates for prevention and perhaps treatment of renal calcium oxalate stones in early stages in humans; further studies are required to confirm the exact mechanism of this effect.

\section{Acknowledgments}

The authors would like to thank the personnel of the research laboratory and animal house of the Birjand University of Medical Sciences. This work was supported by a grant (No. 4316) from the Research Vice Presidency of Birjand University of Medical Sciences.

\section{Conflict of Interest}

The authors declare that they have no conflicts of interest.

\section{Author Contribution}

Reyhane Hoshyar and Maryam Moossavi as corresponding authors contributed to the study design, guides all aspects of study implementation and interpretation of the data as well as critically revised the manuscript for intellectual content. Ahmad Amouzeshi, Seyedeh Zahra Moossavi and Seyed Yoosef Javad Moosavi contributed to data analysis and drafting of the manuscript. Mahmoud Zardast, Mohammad Malekaneh, Sajad Esmaili, Abdolghader Taneh and Nasim Lotfi contributed to study implementation. All authors reviewed and approved the final version of the manuscript.

\section{References}

[1] Sharma D, Dey YN, Sikarwar I, Sijoria R, Wanjari MM, Jadhav AD. In vitro study of aqueous leaf extract of Chenopodium album for inhibition of calcium oxalate and brushite crystallization. Egypt. J Basic Appl Sci. 2016; 3(2):164-71.

[2] Taguchi K, Hamamoto S, Okada A, Unno R, Kamisawa H, Naiki T, et al. Genome-wide gene expression profiling of Randall's plaques in calcium oxalate stone formers. J 
Am. Soc Nephrol. 2017; 28(1):333-47.

[3] Pourmand G, Pourmand B. Epidemiology of stone disease in Iran. Urolithiasis: Springer; 2012. p. 85-7.

[4] Sakhaee K, Maalouf NM, Kumar R, Pasch A, Moe OW. Nephrolithiasis-associated bone disease: pathogenesis and treatment options. Kidney Int. 2011; 79(4):393-403.

[5] Ordon M, Urbach D, Mamdani M, Saskin R, Honey RJDA, Pace KT. The surgical management of kidney stone disease: a population-based time series analysis. J Urol. 2014; 192(5):1450-6.

[6] Scales CD, Lai JC, Dick AW, Hanley JM, van Meijgaard J, Setodji CM, et al. Comparative effectiveness of shock wave lithotripsy and ureteroscopy for treating patients with kidney stones. JAMA Surg. 2014; 149(7):648-53.

[7] Khajavi RadA, Hajzadeh M-A-R, Rajaei Z, Sadeghian M-H, Hashemi N, Keshavarzi Z. Preventive effect of Cynodon dactylon against ethylene glycol-induced nephrolithiasis in male rats. Avicenna J Phytomed. 2011; 1(1):14-23.

[8] Makasana A, Ranpariya V, Desai D, Mendpara J, Parekh V. Evaluation for the antiurolithiasis activity of Launaea procumbens against ethylene glycol-induced renal calculi in rats. Toxicol Rep. 2014; 1:46-52.

[9] Faridi P, Roozbeh J, Mohagheghzadeh A. Ibn-Sina's life and contributions to medicinal therapies of kidney calculi. Iran J Kidney Dis. 2012; 6(5):339.

[10] Saha S, Verma RJ. Inhibition of calcium oxalate crystallization in vitro by an extract of Bergenia ciliate. Arab J Urol. 2013; 11(2):187-92.

[11] Ayenigbara G. Medical utility of Cannabis sativa. IOSR J Pharm. 2012; 2(3):460-3.

[12] Andre CM, Hausman J-F, Guerriero G. Cannabis sativa: the plant of the thousand and one molecules. Front Plant Sci. 2016; 7:19.

[13] Di Marzo V, Piscitelli F. The endocannabinoid system and its modulation by phytocannabinoids. Neurotherapeutics. 2015; 12(4):692-8.

[14] Hoshyar R, Mohaghegh Z, Torabi N, Abolghasemi A. Antitumor activity of aqueous extract of Ziziphus jujube fruit in breast cancer: an in vitro and in vivo study. Asian Pac J Reprod. 2015; 4(2):116-22.

[15] Rajagopal G. A simple colorimetric procedure for estimation of citric acid in urine. Indian J. Exp. Biol. 1984; 22(7):391.

[16] Sriboonlue P, Suwantrai S, Prasongwatana V. An indirect method for urinary oxalate estimation. Clin Chim Acta. 1998; 273(1):59-68.

[17] Türk C, Petřík A, Sarica K, Seitz C, Skolarikos A, Straub M, et al. EAU guidelines on interventional treatment for urolithiasis. Eur. Urol. 2016; 69(3):475-82. 
[18] Karadi RV, Gadge NB, Alagawadi K, Savadi RV. Effect of Moringa oleifera Lam. rootwood on ethylene glycol induced urolithiasis in rats. J Ethnopharmacol. 2006; 105(12):306-11.

[19] Khan SR. Reactive oxygen species, inflammation, and calcium oxalate nephrolithiasis. Transl Androl Urol. 2014; 3(3):256.

[20] Dal Moro F, Mancini M, Tavolini IM, De Marco V, Bassi P. Cellular and molecular gateways to urolithiasis: a new insight. Urol Int. 2005; 74(3):193-7.

[21] Aggarwal KP, Narula S, Kakkar M, Tandon C. Nephrolithiasis: molecular mechanism of renal stone formation and the critical role played by modulators. BioMed Res Int. 2013; 2013.

[22] Comelli F, Bettoni I, Colleoni M, Giagnoni G, Costa B. Beneficial effects of a Cannabis sativa extract treatment on diabetes囚induced neuropathy and oxidative stress. Phytother Res. 2009; 23(12):1678-84.

[23] Bihl G, Meyers A. Recurrent renal stone disease-advances in pathogenesis and clinical management. The Lancet. 2001; 358(9282):651-6.

[24] Golshan A, Hayatdavoudi P, Mousa A, Rad AK, Roshan NM, Abbasnezhad A, et al. Kidney stone formation and antioxidant effects of Cynodon dactylon decoction in male Wistar rats. Avicenna J Phytomed. 2017; 7(2):180.

[25] Saha S, Verma RJ. Antinephrolithiatic and antioxidative efficacy of Dolichos biflorus seeds in a lithiasis rat model. Pharm Biol. 2015; 53(1):16-30.

[26] Saha S, Shrivastav PS, Verma R. Antioxidative mechanism involved in the preventive efficacy of Bergenia ciliate rhizomes against experimental nephrolithiasis in rats. Pharm Biol. 2014; 52(6):712-22. 